\title{
Validation of the short forms of the Pelvic Floor Distress Inventory (PFDI-20), Pelvic Floor Impact Questionnaire (PFIQ-7), and Pelvic Organ Prolapse/Urinary Incontinence Sexual Questionnaire (PISQ-12) in Finnish
}

\author{
Nina Kristiina Mattsson ${ }^{1 *}$ (D), Kari Nieminen ${ }^{2}$, Anna-Mari Heikkinen ${ }^{3}$, Jyrki Jalkanen ${ }^{4}$, Sari Koivurova ${ }^{5}$,
} Marja-Liisa Eloranta ${ }^{6}$, Pia Suvitie ${ }^{7}$ and Anna-Maija Tolppanen ${ }^{8}$

\begin{abstract}
Background: Although several validated generic health-related quality of life instruments exist, disease-specific instruments are important as they are often more sensitive to changes in symptom severity. It is essential to validate the instruments in a new population and language before their use. The objective of the study was to translate into Finnish the short forms of three condition-specific questionnaires (PFDI-20, PFIQ-7 and PISQ-12) and to evaluate their psychometric properties in Finnish women with symptomatic pelvic organ prolapse.

Methods: A multistep translation method was used followed by an evaluation of validity and reliability in prolapse patients. Convergent and discriminant validity, internal consistency and reliability via test-retest were calculated.

Results: Sixty-three patients waiting for prolapse surgery filled the three questionnaires within two weeks. Response rate for each item was high in PFDI-20 and PISQ-12 (99.8 and 98.9\% respectively). For PFIQ-7 response rate was only $60 \%$. In PFIQ-7, six respondents (9.5\%) reached the minimum value of zero showing floor effect. None of the instruments had ceiling effect. Based on the item-total correlations both PFIQ-7 and PFDI-20 had acceptable convergent validity, while the convergent validity of PISQ-12 was lower, $r=0.138-0.711$. However, in this instrument only three questions (questions 6, 10 and 11) had $r<0.3$ while others had $r \geq 0.380$. In the test-retest analysis all the three instruments showed good reliability (ICC 0.75-0.92). Similarly, the internal consistency of the instruments, measured by Cronbach's a, was good (range 0.69-0.96) indicating high homogeneity.

Conclusions: Finnish validated translation of the PFDI-20 and PISQ-12 have acceptable psychometric properties and can be used for both research purposes and clinical evaluation of pelvic organ prolapse symptoms. The Finnish version of PFIQ-7 displayed low response rate and some evidence of a floor effect, and thus its use is not recommended in its current form.
\end{abstract}

Keywords: Pelvic organ prolapse, Symptom questionnaire, Validation, Psychometric evaluation, Reliability, Health related quality of life

\footnotetext{
* Correspondence: nina.mattsson@khshp.fi

${ }^{1}$ Department of Obstetrics and Gynecology, Kanta-Häme Central Hospital,

Hämeenlinna, Finland

Full list of author information is available at the end of the article
} 


\section{Background}

Pelvic floor disorders (PFD) include variable symptoms such as urinary incontinence, feeling of a vaginal bulge, fecal incontinence, and other sensory and emptying abnormalities of the lower urinary and gastrointestinal tracts. The prevalene of women reporting at least one pelvic floor disorder is $23 \%$, which proportion increases with age [1]. These symptoms can have a significant impact on the quality of life and they may cause problems in sexual life [2]. The prevalence of symptomatic pelvic organ prolapse (POP) is estimated to be $3-6 \%$ of adult women and up to $50 \%$ when based upon vaginal examination $[1,3]$. It is necessary to consider not only the underlying anatomical disorder but also women's overall pelvic function and their health-related quality of life when making treatment decisions [3]. For this purpose, condition-specific qualityof-life instruments were developed and published in English 2001 [4, 5]. The Pelvic Floor Distress Inventory (PFDI), the Pelvic Floor Impact Questionnaire (PFIQ), and the Prolapse/Urinary Incontinence Sexual Questionnaire (PISQ) have shown to be psychometrically valid and reliable instruments for measuring the extent to which pelvic floor disorders affect quality of life [4,5]. PFDI investigates the range of POP symptoms and the inconvenience they cause, while PFIQ covers the impact of POP on daily life. PISQ investigates the sexual function of heterosexual women suffering from POP and/or urinary incontinence. The short versions of these three questionnaires have also been validated [6, 7]. PFDI-20 consists of three separate scales: Pelvic Organ Prolapse Distress Inventory (POPDI) of six questions about the inconvenience of the prolapse, Colorectal-Anal Distress Inventory (CRADI) with eight questions concerning difficulties of defecation, and the Urinary Distress Inventory (UDI) with six questions about difficulties in urination. Similarly, the PFIQ-7 consists of three scales, each of them containing seven questions: the Pelvic Organ Prolapse Impact Questionnaire (POPIQ), the Colorectal-Anal Impact Questionnaire (CRAIQ) and the Urinary Impact Questionnaire (UIQ). The short version of PISQ contains 12 questions about sexual activity, satisfaction and problems caused by POP or urinary incontinence.

PFDI-20, PFIQ-7 and PISQ-12 are widely used and they help investigators to evaluate the efficacy of a particular therapy for POP or to compare symptom severity between patients or groups. These disease-specific instruments have been translated and validated in several different countries and in more than ten languages [8-20].

Validated tools for measuring the severity of discomfort of pelvic prolapse and assessing the effectiveness of therapy are not currently available in Finnish. The aim of this study was to translate PFDI-20, PFIQ-7 and PISQ-12 into Finnish and validate these translations among women with symptomatic POP.

\section{Methods}

For the translation process, a group of seven key in-country persons (authors NM, KN, A-MH, JJ, SK, M-LE, PS) were recruited among the board of Finnish Society of Gynecological Surgery. Translation permissions were obtained from the developers of the instruments, Dr. Barber [4] and Dr. Rogers [5]. The translation of the forms was conducted by multistep translation method [21]. Four forward translations of PFDI-20, PFIQ-7 and PISQ were done, two by independent professional translators with experience of translating patient-reported outcome (PRO) measures, and two by $\mathrm{PhD}$ gynecologist experienced in urogynecology. The four translations were tested on a group of four lay persons. Two of the lay persons were urogynecological nurses and two native Finnish-speaking nonprofessionals, one of whom was bilingual (Finnish-English). Each lay person picked the best translation alternatives of the questions or proposed their own alternative wording. One gynecologist (NM) compared lay persons' interpretation and made the final review of the translation. A professional medical translator performed back-translations that were compared to the original questionnaires. The final versions of the translated instruments were approved by the group of key-in country persons (Additional files 1, 2 and 3).

A test-retest analysis was conducted among 63 native Finnish-speaking female patients waiting for surgery for symptomatic POP. The women were recruited from four hospitals: Turku University Hospital, Kuopio University Hospital, Oulu University Hospital and Kanta-Häme Central Hospital. The first three are tertiary university hospitals and the last one is a secondary hospital, all performing urogynecological surgery. The hospitals represent different areas of Finland: western, eastern, northern and southern part of Finland, respectively.

Postal questionnaires including two pre-stamped envelopes were sent to the patients waiting for prolapse treatment. The patients were asked first to fill and return the test questionnaires and then, after 2 weeks, to fill out and return the retest questionnaires. The questionnaires were paired by a code number and analysed anonymously. The participants gave their informed consent by returning the written questionnaires. The study was approved by the Ethical committee of University of Eastern Finland (2014/5), and it followed the ethical standards of the Helsinki Declaration [22].

\section{Statistical and data analysis}

The PFIQ-7, PFDI-20 and PISQ-12 questionnaires and the subscales of PFIQ-7 and PFDI-20 were tested for construct validity and reliability. The average scores in each scale were reported as means and standard deviations, as well as medians and interquartile range due to the skewed distribution of the data. Convergent and discriminant validity were investigated with Spearman's rank order correlation and the corrected item-total 
correlations. Corrected item-total correlations $\geq 0.3$ can be considered as evidence on convergent validity [23]. In addition, response rate, floor and ceiling effects, (i.e., persons obtaining minimum and maximum scores, respectively) were calculated. Overall response rate was defined as the proportion of the patients that returned the two questionnaires in two weeks. Item response rate was defined as the proportion of answered questions in each questionnaire. Reliability was assessed by test-retest analysis and intra-class correlation coefficient (ICC), while the internal consistency was measured with Cronbach's $\alpha$. Cronbach's $\alpha$ was calculated separately for persons with missing data and those who completed all questions in the subscale forms. $\alpha$-values below 0.7 indicate too high heterogeneity, while values above 0.9 indicate too high similarity between items [24]. Thus, the preferred range of $\alpha$ is between 0.7 and 0.9.

Statistical analyses were conducted with Stata 14.0 (Stata Corporation, College Station TX, USA) and IBM SPSS 21.0 (Chicago IL, USA).

\section{Results}

The formation of the study population is shown in Fig. 1. The final sample consisted of 63 women who returned both questionnaires. Twenty-seven of the 63 (42\%) patients who returned both questionnaires were sexually active and completed the PISQ-12. The mean age of the patients was 64.1 years (median 64, range 25-86 years).

The item response rates were $99.8 \%$ for PFDI-20, $60.0 \%$ for PFIQ-7 and $98.9 \%$ for PISQ-12. In PFDI-20 factor scores without any imputations could be calculated in 96.8\% cases for POPDI-6, 98.4\% in cases for CRADI-8 and $100 \%$ in cases for UDI-6 (Table 1). For PFIQ-7, factor scores that could be calculated were $82.5 \%$ of cases for UIQ $-7,77.8 \%$ of cases for CRAIQ-7 and $79.4 \%$ of cases for POPIQ-7.

Floor or ceiling effects were not observed with PFDI-20 or PISQ-12 instruments. There was little evidence of floor effect with subscales of PFIQ-7 (15-17\% responders with minimum value), but no significant floor effect was observed with the summary scale, with four respondents (7\%) having the minimum value of zero (Table 2). Ceiling effects were not observed.

Based on the item-total correlations, both PFIQ-7 and PFDI-20 had acceptable convergent validity (Additional file 4: Table S1 and Additional file 5: Table S2). The correlations were $r=0.601-0.878$ for UIQ-7, $r=0.568-0.907$ for CRAIQ- 7, $r=0.643-0.853$ for POPIQ-7 and $r=0.513-$ 0.865 for the total PFIQ-7. Lower item-total correlations were observed with PFDI-20 $(r=0.309-0.579$ for POPDI-6, $r=0.371-0.486$ for UDI-6, $0.335-0.611$ for CRADI
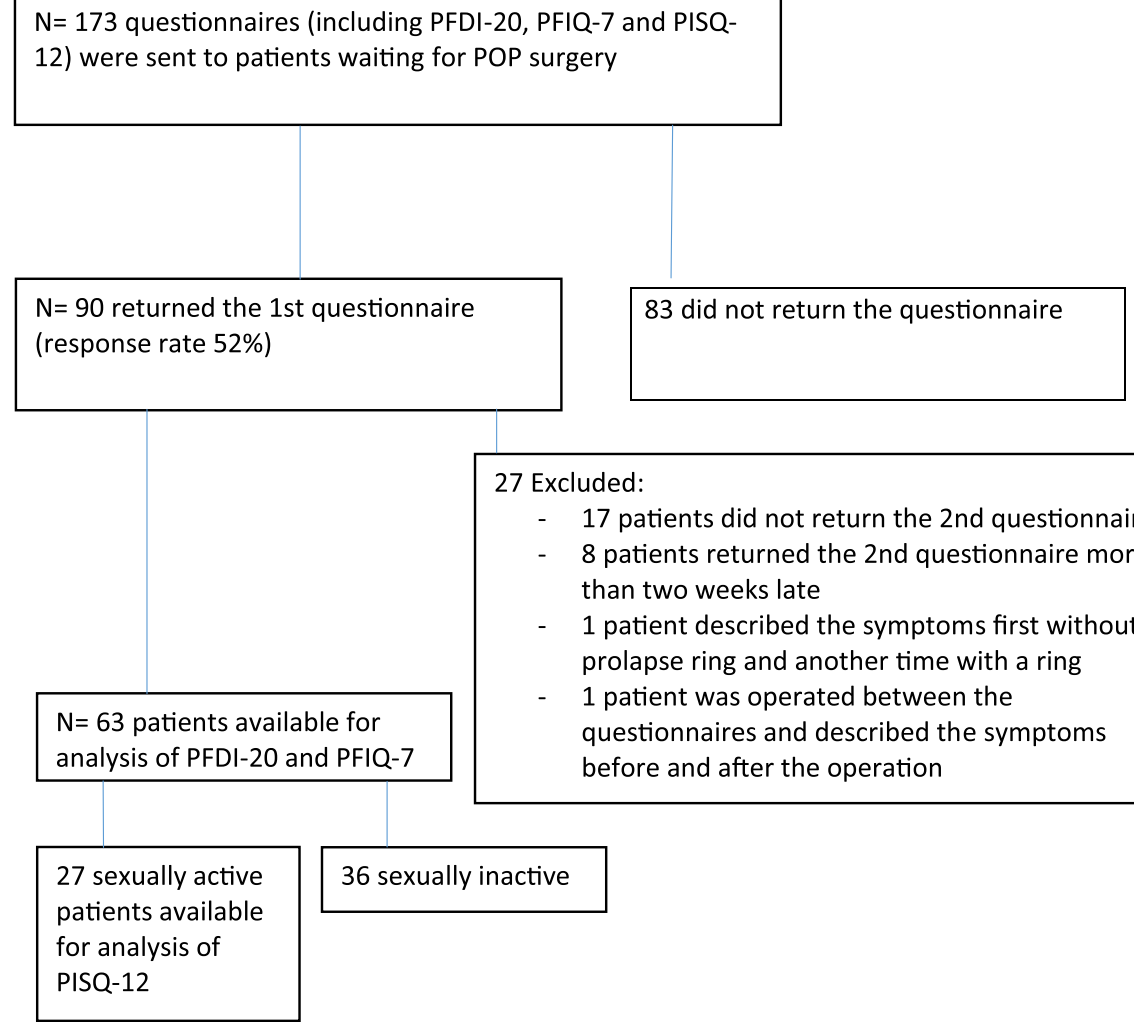

Fig. 1 Flowchart: Inclusion of patient group 





Table 2 Floor and ceiling effects of baseline scores

\begin{tabular}{llll}
\hline $\begin{array}{l}\text { Questionnaire } \\
\text { (scores min-max) }\end{array}$ & $\begin{array}{l}\text { Factor scores } \\
\text { calculated }(n)\end{array}$ & Floor, $n(\%)$ & Ceiling, $n(\%)$ \\
\hline PFDI-20 (0-300) & 63 & $0(0)$ & $0(0)$ \\
POPDI-6 (0-100) & 63 & $0(0)$ & $1(1.6)$ \\
CRADI-8 (0-100) & 63 & $5(8)$ & $0(0)$ \\
UDI-6 (0-100) & 63 & $5(8)$ & $1(1.6)$ \\
PFIQ-7 (0-300) & 58 & $4(7)$ & $0(0)$ \\
POPIQ-7 (0-100) & 58 & $10(17)$ & $0(0)$ \\
CRAIQ-7 (0-100) & 59 & $18(31)$ & $0(0)$ \\
UIQ-7 (0-100) & 59 & $9(15)$ & $2(3.4)$ \\
PISQ-12 (0-48) & 27 & $0(0)$ & $0(0)$ \\
\hline
\end{tabular}

and $r=0.309-0.639$ for the PFDI-20 total score). The lowest convergent validity was observed with PISQ-12, $r=0.138-0.711$ (Additional file 6: Table S3). However, in this instrument only three questions (questions 6,10 and 11) had $r<0.3$, while others had $r \geq 0.380$.

Convergent validity was analyzed by correlation between the three instruments (Table 3). Correlation between PFDI-20 and PFIQ-7 was 0.743, and ranged between 0.492 and 0.929 , including the subscales. In both of these questionnaires, the total score correlated well with their respective subscales. PISQ-12 was negatively correlated with PFDI-20 and PFIQ-7 total scores and subscales $(r=-0.327$ to -0.616$)$. Based on the strenght and direction of the item-total correlations, both PFIQ-7 and PFDI-20 had acceptable convergent validity (Additional file 4: Table S1 and Additional file 5: Table S2).

In the test-retest analysis, all the three instruments showed good reliability (Table 1). Intra-class correlations were strong, varying from 0.75 (PFIQ-7) to 0.92 (PFDI-20). All ICCs were statistically significant $(\mathrm{p}<0.001)$. Similarly, the internal consistency of the instruments, measured by Cronbach's $\alpha$, was between 0.69-0.89 for PISQ-12 and PFDI-20 and its subscales. $\alpha$-values for baseline
PFIQ-7 and its subscales were 0.91-0.96, indicating high homogeneity.

\section{Discussion}

Pelvic organ prolapse itself, its treatment and complications related to it (for example de novo dyspareunia or vaginal mesh exposure following surgery) may have a significant effect on the patient's quality of life. Hence, it is essential to measure the symptoms and HRQOL related to POP with validated instruments, both in clinical practice and research settings. PFDI-20, PISQ-12 and PFIQ-7 have proven to be valid and reliable instruments for measuring symptom inconvenience caused by pelvic organ prolapse and the health-related quality of life $[6,7]$. Until now, their Finnish translations have not been validated. In the present study we have translated these questionnaires in Finnish and assessed the reliability and validity of these Finnish versions among women suffering from symptomatic pelvic organ prolapse in the present study.

The item response rates for PFDI-20 and PISQ-12 were high (99.8 and $98.9 \%$, respectively), whereas the response rate for PFIQ-7 was only 60\%. Ceiling effects were not observed. Floor effect was observed with all three subscales of PFIQ-7, but it was less evident with the summary scales. Cronbach's $\alpha$ of PFIQ was 0.94 and 0.96 indicating that some of the items may be too similar. PFIQ also had the lowest ICC of 0.75 , while the internal consistency of PFDI-20 and PISQ-12 was better (0.92 and 0.87 , respectively). In addition, there was no evidence of too high homogeneity or heterogeneity of individual items in these scales, as indicated by Cronbach's $\alpha$.

Our results show psychometric validity for PFDI-20 questionnaire and are comparable with previous validation studies [8-10]. In our study, PFIQ-7 had some limitations whereas Teleman et al found acceptable psychometric properties in the Swedish version of PFIQ-7 [9]. There was some evidence for a floor effect in our study, although $15-17 \%$ of respondents scored the minimum value with

Table 3 Results from the analysis of convergent validity, i.e. correlation between the three questionnaires (including subscales). Data are given as $r(P)$

\begin{tabular}{|c|c|c|c|c|c|c|c|c|}
\hline Questionnaire & PFIQ-7 & UIQ-7 & CRAIQ-7 & POPIQ-7 & PFDI-20 & POPDI-6 & CRADI-8 & UDI-6 \\
\hline UIQ-7 & $0.929(<0.001)$ & & & & & & & \\
\hline CRAIQ-7 & $0.756(<0.001)$ & $0.621(<0.001)$ & & & & & & \\
\hline POPIQ-7 & $0.847(<0.001)$ & $0.702(<0.001)$ & $0.522(<0.001)$ & & & & & \\
\hline PFDI-20 & $0.743(<0.001)$ & $0.683(<0.001)$ & $0.688(<0.001)$ & $0.565(<0.001)$ & & & & \\
\hline POPDI-6 & $0.565(<0.001)$ & $0.497(<0.001)$ & $0.526(<0.001)$ & $0.459(<0.001)$ & $0.861(<0.001)$ & & & \\
\hline CRADI-8 & $0.623(<0.001)$ & $0.538(<0.001)$ & $0.739(<0.001)$ & $0.406(0.001)$ & $0.821(<0.001)$ & $0.572(<0.001)$ & & \\
\hline UDI-6 & $0.691(<0.001)$ & $0.708(<0.001)$ & $0.492(<0.001)$ & $0.538(<0.001)$ & $0.841(<0.001)$ & $0.624(<0.001)$ & $0.549(<0.001)$ & \\
\hline PISQ-12 & $-0.511(0.006)$ & $-0.506(0.007)$ & $-0.432(0.025)$ & $-0.339(0.084)$ & $-0.616(0.001)$ & $-0.640(<0.001)$ & $-0.496(0.009)$ & $-0.327(0.096)$ \\
\hline
\end{tabular}


each of the subscales, which is considerably less than in the Dutch validation study [10], where the scales of the PFIQ-7 showed floor effects in $44-55 \%$ patients, though the summary score did not. Due et al. reported opposite difficulties with the Danish version of PFIQ-7, with a major ceiling effect and lack of items about health-related quality of life [8]. Thus, some but not all the problems with PFIQ-7 in our study may not be due to cultural reasons. It is not clear why the item response rate of PFIQ-7 was low in our study. In future, it may be reasonable to make another linguistic and cultural validation process for the PFIQ-7 to improve the usefulness of the Finnish translation of this instrument. The PISQ-12 showed acceptable psychometric properties as also evaluated in the Swedish study [9]. Limitation of both studies is the relatively small number of sexually active patients $(N=25$ in reference [9], $N=27$ in our study). Another limitation of PISQ-12 is that it measures the sexual function only among sexually active heterosexual women. Therefore, another instrument to measure pelvic floor disorders' impact on sexual activity for both sexually active and inactive women has been published [25]. This IUGA-revised questionnaire (PISQ-IR) [26] has already been translated and validated into five languages [25, 27-30]. In the future, it would be reasonable to conduct PISQ-IR translation and validation processes also in Finnish to assess its validity in the Finnish context.

The multistep translation method was one of the strengths of this study. The existing evidence supports this approach over the more simple translation - back translation process [21]. The translation and linguistic validation process was conducted in accordance with the guidelines for the translation and cultural adaptation of patient-reported outcome (PRO) measures [31]. We used four different translations and a multi-professional team in the translation process. Cognitive debriefing of the translated versions was done to ensure consistent and accurate interpretation and understanding of the questionnaires among respondents. One of the lay persons was bilingual with English as another home language. The study subjects of this multicenter study represent sufficiently different geographical areas and dialects of the Finnish language. The age distribution (mean 64.1 years, median 64 years) in our study represents the typical age of women suffering from symptomatic pelvic prolapse [3].

One limitation of the study was that we did not record the socioeconomic position of the patients. Hence, it may be possible that, for example, patients with higher education were over-presented in the study. Another, but in our opinion minor drawback in a study of this kind is the overall response rate of only $52 \%$. In the Danish study of Due et al. [8], in which the recruiting process was similar to ours, the response rate was $60 \%$. Reasons for the lower response rate may be the lack of personal contact with the subjects when the forms were sent and the fact that there were no reminders.

\section{Conclusions}

In conclusion, the Finnish versions of PFDI-20 and PISQ-12 are reliable, valid and feasible to evaluate the symptoms and the quality of life in women with pelvic floor disorders. Instead, the Finnish version of PFIQ-7 has some limitations and is not usable in its current form. We suggest that PFDI-20 and PISQ-12 should be used as a patient-reported outcome measure in research and clinical practice.

\section{Additional files}

Additional file 1: PFDI-20 in Finnish. (DOCX $114 \mathrm{~kb}$ )

Additional file 2: PFIQ-7 in Finnish. (DOCX $100 \mathrm{~kb}$ )

Additional file 3: PISQ-12 in Finnish. (DOCX $86 \mathrm{~kb}$ )

Additional file 4: Table S1. Item-total correlations for PFIQ-7 and its subscales. (DOCX $14 \mathrm{~kb}$ )

Additional file 5: Table S2. Item-total correlations for PFDI-20 and its subscales. (DOCX $15 \mathrm{~kb}$ )

Additional file 6: Table S3. Item-total correlations for PISQ-12. (DOCX $12 \mathrm{~kb})$

\section{Abbreviations}

CRADI: Colo-rectal-anal distress inventory; CRAIQ: Colo-rectal-anal impact questionnaire; HRQOL: Health related quality of life; PFDI: Pelvic floor distress inventory; PFIQ: Pelvic floor impact questionnaire; PISQ: Pelvic organ prolapse/ urinary incontinence sexual questionnaire; POP: Pelvic organ prolapse; POPDI: Pelvic organ prolapse distress inventory; POPDI: Pelvic organ prolapse distress inventory; PRO: Patient-reported outcome; UDI: Urinary distress inventory

Acknowledgements

Not applicable.

Funding

This study was partly funded by The Finnish Society of Gynecological Surgery and The Emil Aaltonen Foundation.

Availability of data and materials

The datasets analysed during the current study are available from the corresponding author on reasonable request.

\section{Authors' contributions}

All the authors took part in design of the study protocol, collection, analysis, and interpretation of the data. NM, KN, and A-MT had the main responsibility of preparing the manuscript, but the other authors carefully revised the manuscript. All the authors have reviewed the final version of the manuscript and approve it for publication.

\section{Competing interests}

The authors declare that they have no competing interests.

\section{Consent for publication}

Not applicable.

\section{Ethics approval and consent to participate}

The Research Ethics Committee of the Northern Savo Hospital District approved the study on 20th of May 2014: (Reference number 5//2014). The participates gave their consent by answering the questionnaires. The study data was collected and analysed anonymously. 


\section{Endnotes}

The Finnish versions of PFDI-20 and PISQ-12 are reliable, valid and feasible to evaluate the symptoms and the quality of life in women with pelvic floor disorders and should be used as a patient-reported outcome measure in research and clinical practice.

\section{Publisher's Note}

Springer Nature remains neutral with regard to jurisdictional claims in published maps and institutional affiliations.

\section{Author details}

'Department of Obstetrics and Gynecology, Kanta-Häme Central Hospital, Hämeenlinna, Finland. ²Department of Obstetrics and Gynecology, Tampere University Hospital, Tampere, Finland. ${ }^{3}$ Terveystalo, Kuopio, Finland. ${ }^{4}$ Department of Obstetrics and Gynecology, Central Finland Hospital District, Jyväskylä, Finland. ${ }^{5}$ Department of Obstetrics and Gynecology, Oulu University Hospital, Oulu, Finland. ${ }^{6}$ Department of Obstetrics and Gynecology, Kuopio University Hospital, Kuopio, Finland. 'Department of Obstetrics and Gynecology, Turku University Hospital and University of Turku, Turku, Finland. ${ }^{8}$ Research Centre for Comparative Effectiveness and Patient Safety (RECEPS) and School of Pharmacy, University of Eastern Finland, Kuopio, Finland.

\section{Received: 14 September 2016 Accepted: 5 April 2017}

\section{Published online: 02 May 2017}

\section{References}

1. Nygaard I, Barber MD, Burgio KL, Kenton K, Meikle S, Schaffer J, et al. Prevalence of symptomatic pelvic floor disorders in US women. JAMA. 2008:300(11):1311-6.

2. Jelovsek JE, Barber MD. Women seeking treatment for advanced pelvic organ prolapse have decreased body image and quality of life. Am J Obstet Gynecol. 2006;194(5):1455-61

3. Barber MD, Maher C. Epidemiology and outcome assessment of pelvic organ prolapse. Int Urogynecol J. 2013;24(11):1783-90.

4. Barber MD, Kuchibhatla MN, Pieper CF, Bump RC. Psychometric evaluation of 2 comprehensive condition-specific quality of life instruments for women with pelvic floor disorders. Am J Obstet Gynecol. 2001;185(6):1388-95.

5. Rogers RG, Kammerer-Doak D, Villarreal A, Coates K, Qualls C. A new instrument to measure sexual function in women with urinary incontinence or pelvic organ prolapse. Am J Obstet Gynecol. 2001;184(4):552-8.

6. Barber MD, Walters MD, Bump RC. Short forms of two condition-specific quality-of-life questionnaires for women with pelvic floor disorders (PFDI-20 and PFIQ-7). Am J Obstet Gynecol. 2005;193(1):103-13.

7. Rogers RG, Coates KW, Kammerer-Doak D, Khalsa S, Qualls C. A short form of the pelvic organ prolapse/urinary incontinence sexual questionnaire (PISO-12). Int Urogynecol J Pelvic Floor Dysfunct. 2003:14(3):164-8. discussion 168.

8. Due U, Brostrom S, Lose G. Validation of the pelvic floor distress inventory-20 and the pelvic floor impact questionnaire-7 in danish women with pelvic organ prolapse. Acta Obstet Gynecol Scand. 2013;92(9):1041-8.

9. Teleman P, Stenzelius K, lorizzo L, Jakobsson U. Validation of the swedish short forms of the pelvic floor impact questionnaire (PFIQ-7), pelvic floor distress inventory (PFDI-20) and pelvic organ prolapse/urinary incontinence sexual questionnaire (PISQ-12). Acta Obstet Gynecol Scand. 2011;90(5):483-7.

10. Utomo E, Blok BF, Steensma AB, Korfage IJ. Validation of the pelvic floor distress inventory (PFDI-20) and pelvic floor impact questionnaire (PFIQ-7) in a dutch population. Int Urogynecol J. 2014:25(4):531-44.

11. Yoshida M, Murayama R, Ota E, Nakata M, Kozuma S, Homma Y. Reliability and validity of the Japanese version of the pelvic floor distress inventory-short form 20. Int Urogynecol J. 2013;24(6):1039-46.

12. Bilgic Celik D, Beji NK, Yalcin O. Turkish adaptation of the short form of the pelvic organ prolapse/urinary incontinence sexual function questionnaire (PISQ-12): a validation and reliability study. Neurourol Urodyn. 2013;32(8):1068-73.

13. Santana GW, Aoki T, Auge AP. The Portuguese validation of the short form of the pelvic organ prolapse/urinary incontinence sexual questionnaire (PISQ-12). Int Urogynecol J. 2012;23(1):117-21.

14. Sanchez-Sanchez B, Torres-Lacomba M, Yuste-Sanchez MJ, Navarro-Brazalez B, Pacheco-da-Costa S, Gutierrez-Ortega C, et al. Cultural adaptation and validation of the pelvic floor distress inventory short form (PFDI-20) and pelvic floor impact questionnaire short form (PFIQ-7) spanish versions. Eur J Obstet Gynecol Reprod Biol. 2013;170(1):281-5.

15. Arouca MA, Duarte TB, Lott DA, Magnani PS, Nogueira AA, Rosa-E-Silva JC, et al. Validation and cultural translation for Brazilian Portuguese version of the Pelvic Floor Impact Questionnaire (PFIQ-7) and Pelvic Floor Distress Inventory (PFDI-20). Int Urogynecol J. 2016;27(7):1097-106.

16. de Tayrac R, Deval B, Fernandez H, Mares P, Institute MR. Development of a linguistically validated French version of two short-form, condition-specific quality of life questionnaires for women with pelvic floor disorders (PFDI-20 and PFIQ-7). J Gynecol Obstet Biol Reprod (Paris). 2007;36(8):738-48.

17. Pons EM, Clota PM, Aguilon GM, Zardain PC, Alvarez RP. Questionnaire for evaluation of sexual function in women with genital prolapse and/or incontinence. Validation of the spanish version of "pelvic organ prolapse/urinary incontinence sexual questionnaire (PISQ-12)". Actas Urol Esp. 2008;32(2):211-9.

18. Yoo EH, Jeon MJ, Ahn KH, Bai SW. Translation and linguistic validation of Korean version of short form of pelvic floor distress inventory-20, pelvic floor impact questionnaire-7. Obstet Gynecol Sci. 2013;56(5):330-2.

19. Zhu L, Yu S, Xu T, Yang X, Lu Y, Lang J. Validation of the chinese version of the pelvic organ prolapse/urinary incontinence sexual questionnaire short form (PISQ-12). Int J Gynaecol Obstet. 2012;116(2):117-9.

20. Grigoriadis T, Athanasiou S, Giannoulis G, Mylona SC, Lourantou D, Antsaklis A. Translation and psychometric evaluation of the greek short forms of two condition-specific quality of life questionnaires for women with pelvic floor disorders: PFDI-20 and PFIQ-7. Int Urogynecol J. 2013:24(12):2131-44.

21. Acquadro C, Conway K, Hareendran A, Aaronson N. European regulatory issues and quality of life assessment (ERIQA) group. Literature review of methods to translate health-related quality of life questionnaires for use in multinational clinical trials. Value Health. 2008;11(3):509-21.

22. World Medical Association. World medical association declaration of helsinki: ethical principles for medical research involving human subjects. JAMA. 2013:310(20):2191-4.

23. Kline $P$. The handbook of psychological testing. 2nd ed. London: Routledge; 1999.

24. Cristobal E, Flavian C, Guinaliu M. Perceived e-service quality (PeSQ): measurement validation and effects on consumer satisfaction and web site loyality. Manag Serv Qual. 2007;17(3):317-04.

25. El-Azab AS, Ghoniem GM, Leu SY, Nguyen DV. Arabic validation of the pelvic organ prolapse/incontinence sexual questionnaire, IUGA-revised (PISQ-IR). Int Urogynecol J. 2015;26(8):1229-37.

26. Rogers RG, Rockwood TH, Constantine ML, Thakar R, Kammerer-Doak DN, Pauls RN, et al. A new measure of sexual function in women with pelvic floor disorders (PFD): the pelvic organ prolapse/incontinence sexual questionnaire, IUGA-revised (PISQ-IR). Int Urogynecol J. 2013; 24(7):1091-103.

27. Fatton B, Hermieu JF, Cour F, Wagner L, Jacquetin B, de Tayrac R. French language validation of the pelvic organ prolapse/urinary incontinence sexual questionnaire - IUGA revised (PISQ-IR). Prog Urol. 2013;23(17):1464-73.

28. Tomoe $H$, Inoue M, Kimoto $Y$, Nagao K, Homma Y, Takahashi $S$, et al. Linguistic validation of japanese version of prolapse/urinary incontinence sexual questionnaire, IUGA-revised (PISQ-IR). Nihon Hinyokika Gakkai Zasshi. 2014;105(3):102-11.

29. Trutnovsky G, Nagele E, Ulrich D, Aigmuller T, Dorfler D, Geiss I, et al. German translation and validation of the Pelvic Organ Prolapse/Incontinence Sexual Questionnaire-IUGA revised (PISQ-IR). Int Urogynecol J. 2016;27(8):1235-44. doi: 10.1007/s00192-016-2969-9.

30. Wang H, Lau HH, Hung MJ, Huang WC, Zheng YW, Su TH. Validation of a mandarin chinese version of the pelvic organ prolapse/urinary incontinence sexual questionnaire IUGA-revised (PISQ-IR). Int Urogynecol J. 2015:26(11):1695-700.

31. Wild D, Grove A, Martin M, Eremenco S, McElrov S, Verjee-Lorenz A, et al. Principles of good practice for the translation and cultural adaptation process for patient-reported outcomes (PRO) measures: report of the ISPOR task force for translation and cultural adaptation. Value Health. 2005:8(2):94-104. 